\title{
Vers une génétique de la critique : le cas de Georges Poulet
}

\section{Colton Valentine}

\section{(2) OpenEdition}

1 Journals

\section{Édition électronique}

URL : http://journals.openedition.org/genesis/2814

DOI : 10.4000/genesis.2814

ISSN : 2268-1590

\section{Éditeur :}

Presses universitaires de Paris Sorbonne (PUPS), Société internationale de génétique artistique littéraire et scientifique (SIGALES)

\section{Édition imprimée}

Date de publication : 4 juin 2018

Pagination : 173-181

ISBN : 979-10-231-0604-6

ISSN : 1167-5101

\section{Référence électronique}

Colton Valentine, "Vers une génétique de la critique : le cas de Georges Poulet », Genesis [En ligne],

46 | 2018, mis en ligne le 01 juin 2019, consulté le 10 décembre 2020. URL : http://

journals.openedition.org/genesis/2814; DOI : https://doi.org/10.4000/genesis.2814 


\title{
Vers une génétique de la critique : le cas de Georges Poulet
}

\author{
Colton Valentine
}

Comme nous l'avons déjà fait par le passé, dans les numéros 16 et 34, nous publions ici, tel quel, un remarquable travail de validation rendu par un étudiant au terme d'un séminaire de critique génétique. On vérifie une fois encore que, dans notre domaine, la valeur n'attend pas le nombre des années.

Q u'est-ce qu'une critique génétique de la critique ? D'emblée, définissons la critique génétique comme la collecte et la mise en ordre des avant-textes, mais également et plus récemment des après-textes ${ }^{1}$, une manière de saisir la signification plus profonde ou au moins plus complète d'un ouvrage. L'analyse génétique d'un poème de Stéphane Mallarmé retrace, par exemple, sa métamorphose à travers ses brouillons, sa mise au net, et ses variantes publiées. On peut toutefois se demander s'il est légitime d'adapter cette procédure à la critique de Maurice Blanchot portant sur Mallarmé. Il ne s'agit pas simplement de ramasser et d'ausculter les brouillons avec une démarche identique, car le genre de la source a transformé la génétique en métacritique. Une étude du poème «Le Guignon», comme celle récemment faite par Marc Dominicy ${ }^{2}$, établit une distance entre le dispositif (critique) et l'objet d'étude (poétique), mais une enquête sur L'Espace littéraire ne bénéficierait pas de cette démarcation claire et nette. Si l'on accepte la déclaration de Louis Hay selon laquelle «la génétique appartient au système général des études littéraires dont elle partage le territoire», l'ouvrage de Blanchot et l'ouvrage génétique qui l'analyse, se situeraient tous les deux à l'intérieur d'un même système ou au même niveau conceptuel ${ }^{3}$.

Dans ce travail, on se propose d'explorer les conséquences méthodologiques et analytiques d'une telle génétique de la critique, en abordant l'étude de cas de Georges Poulet. Écrivain belge et membre de l'École de Genève, Poulet est devenu célèbre grâce à son chef-d'œuvre Études sur le temps humain, une série de volumes qui se composent de diverses monographies interrogeant le modèle de temps porté par différents écrivains et penseurs ${ }^{4}$. Cette structure charpente la plupart de ses ouvrages, notamment Les Métamorphoses du cercle, La Conscience critique, et La Pensée indéterminée, des textes qui se caractérisent tous par la même forme, celle d'une assemblée de monographies qui retracent l'évolution d'un sujet central. Prenant en considération cette tendance structurale, on se focalisera sur un dossier d'avant-textes qui préfigure et esquisse les chapitres concernant Gustave Flaubert. Premièrement, on cherchera à codifier les différentes étapes de ce que l'on appellera la «procédure critique» de Poulet, autrement dit la façon, inductive et systématique, dont il procède à partir d'une lecture de l'œuvre flaubertienne jusqu'à la rédaction de ses propres chapitres critiques. À travers une série de fiches de citations et de brouillons, Poulet collige, catégorise, et intègre dans son écriture les citations de Flaubert qu'il juge

1. Voir «Réécritures et variation : pour une génétique linguistique et textuelle» par Jean-Michel Adam dans Génétique de la production écrite et linguistique, où il élargit le champ des supports jusqu'à inclure les versions ainsi nommées et stables du texte après sa publication. L'édition récente de Genesis, sous-titrée «Après le texte. De la réécriture après publication», se consacre spécifiquement à ces développements.

2. Marc Dominicy, «Retoucher cette pièce dans le goût d'autrefois : pourquoi Mallarmé a-t-il réécrit "Le Guignon"?», Genesis "Après le texte. De la réécriture après publication », vol. LXIV, 2017, p. 97-108. 3. Louis Hay, «Qu'est-ce que la critique génétique ?», ITEM, mis en ligne le 9 décembre 2008, <http://www.item.ens.fr/index.php?id=384032> (consulté en décembre 2017).

4. Ces enquêtes furent menées au fonds Georges Poulet à Berne, où, grâce à l'aide de Stéphanie Cudré-Mauroux, la consultation fructueuse des dossiers de lecture et des brouillons de Poulet, ainsi que d'autres documents supplémentaires comme des articles de jeunesse, sa correspondance, et sa thèse de doctorat a été rendue possible. 
pertinentes pour l'ouvrage en composition 5 . Deuxièmement, on constatera que la mise en lumière de ces démarches élucide et justifie certains choix stylistiques et méthodologiques propres à l'écrivain belge qui sont souvent considérés comme déroutants, voire même rebutants. Finalement, on explorera la façon dont la logique interne de la praxis de Poulet autorise une telle approche génétique, et dans quelle mesure elle pourrait fournir un socle théorique pour d'autres études de cet acabit.

Avant de plonger dans la masse accablante des brouillons de Poulet, il convient d'esquisser brièvement sa formation académique et la méthode critique induite. Né en 1902 à Chênée, Poulet a suivi des études de littérature et de philosophie à l'université de Liège. Pendant la rédaction de sa thèse de doctorat intitulée «Les Relations entre les personnages dans le roman balzacien», il a simultanément écrit et publié un vaste corpus d'articles critiques et un roman sous le nom de Georges Thialet. Ensuite, il a commencé sa carrière professorale à l'université d'Édimbourg, un lieu assez excentré où il a rédigé le premier volume de son célèbre ouvrage Études sur le temps humain. Grâce à ce texte, Poulet a quitté la périphérie du champ académique, car le succès rencontré lui a permis d'accéder à un poste prestigieux à l'université Johns Hopkins aux États-Unis et, par la suite, à l'université de Zurich et à l'université de Nice. La «critique d'identification» ou «critique de conscience» que Poulet emploie est toujours associée à «l'École de Genève», un groupe qui compte dans ses rangs les critiques littéraires Marcel Raymond, Albert Béguin, Jean Rousset et Jean Starobinski. Pourtant, le nom, donné ultérieurement par J. Hillis Miller, a mené à des confusions considérables en ce qui concerne la nature du groupe. Poulet n'a jamais enseigné à l'université de Genève, et «L'École» était plus la manifestation d'une collaboration bricolée qu'un cénacle codifié et constitué autour d'une seule doctrine, d'une seule approche critique 6 .

En effet, diverses approches analytiques ont nourri la pensée de Poulet, dont seulement certaines furent partagées avec ses collaborateurs. Il fut inspiré d'abord par la philosophie d'Henri Bergson, qu'il a découverte à travers l'œuvre d'Albert Thibaudet, ensuite par la rencontre avec la psychanalyse de Sigmund Freud, une conceptualité féconde mais sans doute relue par l'École à ses propres fins critiques. Néanmoins, l'influence la plus importante pour Poulet fut la phénoménologie d'Edmund Husserl, une philosophie qu'il a adaptée aux études littéraires, afin de vanter et de théoriser l'expérience immanente de la lecture. D'après les mots de Terry Eagleton, il s'agit d'une lecture d'Einfühlung ou de l'identification du critique qui se charge de la reconstruction du Lebenswelt ou du «monde vital» du texte 7 . Ce Lebenswelt est défini avant tout par son aspect total et uni, pourtant, le fond de cette unité n'est ni biographique, ni psychologique, mais métaphorique et subjectif, et décrit par Antoine Compagnon comme le monde des «structures profondes d'une vision du monde 8 ». Ce propos tout à fait unique dans le cas de Poulet, synthétisé au carrefour de plusieurs courants critiques, le mettra en opposition directe avec les vagues du structuralisme qui ont émergé dans les années suivant la parution de son ouvrage Études sur le temps humain.

Forts de ces informations générales, plongeons dans la richesse des manuscrits de Poulet, tout en rappelant l'aboutissement de cette étude, qui est notamment de saisir un rapport entre ce corpus et son propos critique. Quant à l'organisation des avant-textes, elle est facilitée

5. Deux pierres de touche essentielles pour ces investigations sont les articles «Critiquer, c'est se souvenir» par Stéphanie Cudré-Mauroux et «La mémoire du critique: Georges Poulet et Marcel Proust»par Guillaume Perrier. Stéphanie Cudré-Mauroux livre une vue d'ensemble des trésors des manuscrits de Poulet et de leur valeur quant à l'élucidation de sa pensée, bien qu'elle n'inscrive pas explicitement ses enquêtes dans le cadre méthodologique de la génétique. De l'autre côté, Guillaume Perrier opère expressément avec cette démarche, néanmoins son aboutissement critique tient dans la relation entre Poulet et Proust, sans prétention de trop extrapoler les enjeux méthodologiques d'une génétique de la critique.

6. Une excellente synthèse de l'histoire et de la consécration fictive de l'École se trouve dans Theoretical Schools and Circles in the Twentieth Century Humanities, où Oliver Pot trace les divers courants théoriques du groupe, son rapprochement de la nouvelle critique en France, et sa consécration lors d'un congrès à Paris, qui a eu lieu le 26 juillet 1963 . Olivier Pot constate que les œuvres de ces individus témoignent d'un moment charnière, où la forme et la signification pourraient être pensées ensemble avant leur séparation dans les courants du structuralisme et du déconstructionnisme. Pourtant, il en diminue l'héritage, comme étant principalement une «critical fiction» qui fascine et donne du plaisir (p. 204).

7. Terry Eagleton, Literary Theory: An Introduction, 1983, Anniversary Ed. Malden, MA, Backwell, 2008, p. 51.

8. Antoine Compagnon, Le Démon de la théorie, Paris, Éditions du Seuil, 1998 , p. 74. 
par la démarche systématique de Poulet, ainsi que par la conservation consciencieuse des documents au fonds Georges Poulet ${ }^{9}$. Les textes les plus appropriés pour une critique génétique se trouvent dans la catégorie $\mathrm{A}$ « Dossiers de notes et manuscrits », où deux types d'avant-texte prédominent : les fiches de citations qui rassemblent et annotent plusieurs passages textuels, et les brouillons qui esquissent les chapitres et les sous-chapitres des ouvrages. Ces deux types de documents impliquent déjà une certaine chronologie quant aux étapes du travail critique : la collecte des citations, leur catégorisation et leur mise en ordre, et finalement leur incorporation dans la rédaction du texte critique. Même dans les formes des avant-textes, deux accents apparaissent : l'un pourtant sur la singularité de l'écrivain comme objet d'étude, le second, sur le haut statut et la primauté de la citation.

Une lecture plus attentive des fiches de citations permet de les différencier en deux catégories principales. La première catégorie, rédigée pour la plupart avec une machine à écrire, indique sur chaque page le nom de l'auteur en question, ainsi que le titre et l'édition de l'ouvrage. Chaque citation est précédée par le numéro de tome, s'il s'agit d'une compilation, ainsi que le numéro de page. Par exemple, dans la fiche intitulée «GUSTAVE FLAUBERT : CORRESPONDANCE», la mention «À Louise Colet, 26 août 1846. (Conard, I, 270, Charp. I, 137)» préface la citation, dont certaines phrases sont mises en majuscules : «Oui, j'ai UN DÉGÔUT PROFOND DU JOURNAL, C'EST-À-DIRE DE L'ÉPHÉMÈRE, DU PASSAGER, de ce qui est important aujourd'hui et de ce qui ne le sera pas

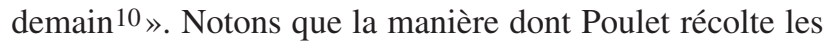
citations dans ces fiches débouche sur deux effets presque paradoxaux, la conservation stricte de la référence bibliographique coexistant avec l'enlèvement radical du contexte de la source. Les mots eux-mêmes s'éloignent de leur point d'origine, un phénomène corroboré par la mise en avant des phrases encore plus isolées, à travers les majuscules.

L'étape suivante de la procédure critique de Poulet apparaît à l'intérieur d'une couche d'ajouts en couleur, écrits à la main, sur ces mêmes fiches. On peut différencier trois types de marques : le soulignement de certains mots dans les citations, le chiffrage des citations, et l'ajout des termes thématiques, comme «illusion» ou «avenir», dans les marges 11 . Dans la fiche citée ci-dessus, les deux mots «L'ÉPHÉMÈRE » et «PASSAGER » sont soulignés en bleu, et un chiffre est accordé à chaque passage pris de la correspondance, ne respectant visiblement pas l'ordre chronologique, et avec des répétitions des mêmes chiffres. Les termes thématiques ou conceptuels qui partagent les marges avec ces chiffres sont écrits de trois façons. «Histoire», «illusion», et «souv. hist.» (abréviation, comme l'on verra plus tard, de «Souvenir Histoire») sont en noir et encadrés, «cause» et «rumination» sont en vert, et «avenir» est en bleu12. Bien que la signification de chaque type de marque reste difficile à saisir, on peut constater que deux types d'indexation, celle des chiffres et celle des thèmes, coexistent et jouent des rôles différents. Dans un sens plus général, la manière systématique dont chaque fiche est assemblée, réexaminée et annotée, témoigne d'une procédure critique fondée sur la relecture, sur une sorte de métabolite ou digestion progressive des sources primaires.

Le rôle de ces classifications se clarifie quand on regarde la deuxième catégorie de fiches de citation : les feuillets qui portent le titre d'un sujet ou d'un concept. Toujours dans la catégorie A-1, ces fiches se distinguent de celles dans les «dossiers thématiques» d'A-3, car elles restent monographiques, recueillant encore des extraits appartenant à un

9. Une description et une table des matières du patrimoine des archives se trouvent sur le site internet du Fonds : < http://ead.nb.admin.ch/html/ poulet_0.html > (consulté en décembre 2017). Tout comme la structure monographique des ouvrages publiés, la majorité des avant-textes sont fédérés dans des dossiers traitant un écrivain singulier (A-1). Les autres sont des «dossiers composites» (A-2) qui sondent un groupe d'écrivains, et des «dossiers thématiques » (A-3) qui examinent un concept plus vaste, comme celui de «Cercles» ou de «Littérature anglaise XIX ${ }^{\mathrm{e}}$ siècle».

10. Fonds Georges Poulet, conservé aux Archives littéraires suisses, Boîte 4, A-1-40.

11. Dans «Critiquer, c'est se souvenir», d'après les mots de Poulet luimême, Stéphanie Cudré-Mauroux appelle ces termes un " "attirail" de catégories, temps, espace, cause, nombre et conscience de soi », signalant le fait qu'ils sont présélectionnés et appliqués après coup à l'amas d'extraits parcourus (p. 110).

12. Malheureusement, les fiches ne portent ni le titre de l'ouvrage critique pour lequel elles furent prévues, ni une éventuelle date qui faciliterait cette attribution. Néanmoins, les termes thématiques qui ont été mis dans les marges prévoient, pour le lecteur assidu, le destin des citations. Dans ce dossier, par exemple, la répétition fréquente du terme «centre» implique que les fiches furent composées pour Les Métamorphoses du cercle, un ouvrage qui se focalise sur l'opposition et l'interaction des termes «le centre» et «la périphérie». Une codicologie des avant-textes de Poulet dépend ainsi moins du contenu en filigrane, mais plus des concepts de l'attirail qu'il a jugé pertinent de retracer. 
seul écrivain. Leur rôle se clarifie dans le dossier sur Flaubert, car on trouve des fiches intitulées «Souvenir-Histoire» et «Expansion, diffusion», ce qui correspond directement à l'attirail des termes thématiques mis dans les marges des exemplaires du premier type de fiches. Une comparaison des citations étiquetées par le terme «souv. hist» avec celles qui apparaissent dans la fiche «Souvenir-Histoire» implique l'idée que les extraits furent probablement recopiés du premier groupe de fiches et systématisés dans le deuxième, selon les thèmes en question. Un effet notoire du transfert est l'escamotage continuel des contextes originaux des citations, car, même si les références bibliographiques se conservent, les extraits de tout un éventail d'ouvrages flaubertiens se brouillent. Dans «Souvenir-Histoire», on découvre une citation de la correspondance de Flaubert à côté d'une citation de Madame Bovary, sans aucune indication sur le fait que le genre du texte modifie le rapport existant entre l'extrait et le concept en question. Ensuite, cet amalgame subit une phase supplémentaire de la digestion critique, dont témoigne une nouvelle couche d'ajouts, relativement moins systématiques et moins prédominants que ceux du premier groupe de fiches. Dans «Souvenir-Histoire», par exemple, quelques phrases sont encerclées et reliées avec des lignes vertes, d'autres sont soulignées en bleu, et une nouvelle marque apparaît : la biffure de citations complètes.

De façon subtile, ces biffures préfigurent l'existence d'un troisième type d'avant-texte : les brouillons où Poulet rédige les premières versions des sous-chapitres d'un ouvrage. Contrairement aux fiches où il amasse et annote les mots d'autres écrivains, ces brouillons se composent principalement de ses propres mots. Pourtant, ces documents représentent plus une continuation qu'une rupture avec les autres avant-textes, car ils portent souvent les mêmes titres thématiques qui nomment le deuxième groupe de fiches de citations. De plus, les citations intégrées dans leur fond sont très souvent celles qui furent biffées dans la fiche correspondante, ce qui témoigne d'un lien direct entre les deux étapes de rédaction. Très réduite, ne dépassant jamais une ou deux pages, la longueur des brouillons équivaut à celle des sous-parties dans les ouvrages publiés, une proximité avec la mise au net corroborée par la pénurie relative des marques de relecture. Certes, quelques petites biffures et ajouts indiquent le souhait de nuancer encore davantage le style de l'écriture. Mais l'on ne déniche pas d'avalanche de notes de régie, ni de mandats adressés à soi-même, comme «à retoucher» ou «à rédiger», qui remplissent très souvent les marges d'un brouillon. La netteté relative de ces brouillons indique que, pour Poulet, la majeure partie du travail interprétatif est faite dans les phases de la récolte et de la digestion de citations. La rédaction proprement dite devient, avant tout, une entreprise de synthèse de cette pâture.

Ces trois formes d'avant-texte dans le dossier sur Flaubert permettent une première esquisse de la procédure critique de Poulet. Quand il vise à composer un chapitre monographique à propos d'un écrivain, il avance en suivant trois étapes principales : l'assemblage des citations de chaque ouvrage de cet écrivain, la classification de ces citations selon des termes ou concepts abstraits, et la rédaction de courts textes prenant chacun comme sujet un de ces concepts. Indubitablement, cette démarche varie beaucoup selon l'ouvrage et le dossier en question, et celui traitant de Flaubert ne fournit qu'une étude de cas à nuancer et à approfondir à travers une enquête plus vaste ${ }^{13}$. En commençant par le rôle des chiffres comme deuxième forme de catégorisation, et jusqu'aux étapes intermédiaires entre les brouillons des sous-chapitres et les chapitres des ouvrages complets, beaucoup de travail reste à faire dans la phase plus scientifique de la génétique textuelle. Pourtant, prenons le risque d'avancer vers une phase plus herméneutique, et de lancer quelques gestes interprétatifs qui lient ce croquis de la procédure de la critique d'identification à ses principes et conclusions, tels que Poulet les a codifiés.

Rebondissons ainsi sur l'escamotage progressif du contexte des citations quand elles passent du premier type de fiche au deuxième, afin de s'intégrer finalement dans le fonds des brouillons. Ici, un germe se tapit, celui d'une idiosyncrasie propre à la critique d'identification : le mélange des données textuelles sans démarcation nette et claire entre leur genre ou espèce. Cette tendance se manifeste pleinement dans le chapitre XV du premier volume de l'ouvrage Études sur le temps humain, qui sonde le modèle de temps flaubertien. Poulet choisit de commencer le chapitre

13. Les travaux effectués par Guillaume Perrier sur le dossier de Proust esquissent un modèle similaire. Même si Guillaume Perrier ne plonge pas dans les détails des fiches, il affirme que le travail préparatoire de Poulet «consiste donc à amasser un très grand nombre de citations » et que ces citations sont catégorisées avec «un codage - numéros, traits de couleurs, etc. - qui permettra de les utiliser au moment de la rédaction. » 
par une citation de sa correspondance concernant «la lueur d'un enthousiasme» qu'il perçoit de temps en temps dans ces «grands jours de soleil14». Immédiatement après, il cite Voyage en Pyrénées et en Corse et le poème en prose Tentation, tout en expliquant que ces moments de réflexion «constituent dans la vie de Flaubert une série de cimes rayonnantes autour desquelles œuvres, pensée, existence, tout se dispose ${ }^{15} »$. L'œuvre et la vie de Flaubert s'assimilent l'une à l'autre, enlevant implicitement toute différentiation générique entre une lettre intime, un récit de voyage, et la littérature proprement dite, pour que les trois textes servent pareillement comme «le point de départ chez Flaubert 16,17 ». Ce procédé est appliqué de la même façon concernant la disparition de marquage générique qui a lieu quand les citations sont transférées des fiches traitant les ouvrages flaubertiens comme «CORRESPONDANCE» vers les fiches thématiques comme «Souvenir-Histoire». De cette manière, dans ces avant-textes se dissimulent en filigrane les germes de ce principe au sein de la critique d'identification. Cependant, la relation causale entre la procédure dont témoignent les avant-textes et le propos des ouvrages publiés reste difficile à saisir : soit Poulet nie a priori la valeur des bornes génériques et organise ses fiches afin de se conformer à ce postulat, soit il compose les fiches sans postulat préconçu et finit, presque par hasard, par lancer des études qui déconstruisent les bornes. Les valeurs et les limites d'une génétique de la critique émergent également de cette ambiguïté : elle réussit à mettre en relation une procédure et un propos, mais elle reste incapable de préciser la causalité entre les deux objets.

Certes, cette tendance inductive à mettre en première position les citations et à faire disparaître les frontières entre leurs sources prédomine dans l'œuvre de Poulet, mais un autre penchant déductif apparaît quand une gamme de supports plus étendue est examinée ${ }^{18}$. Un feuillet qui mérite cet élargissement, intitulé «Caractères temporels des romans de Flaubert», comprend de brèves synthèses sur la temporalité dans les ouvrages de Flaubert en question, comme par exemple :

Salammbô ou le roman absolument historique - Durée qui est celle d'une civilisation - L'imagination intégrale d'un passé dont il ne reste rien - Effort inductif à partir d'un minimum de données - caractère éternel d'un ensemble rêvé, de la beauté retrouvée ${ }^{19}$.
Un abrégé similaire est proposé pour Madame Bovary, L'Éducation sentimentale, Bouvard et Pécuchet, Tentation de saint Antoine, et Trois Contes, le dernier décrit de façon presque épigrammatique comme «Synthèse des différentes durées. » Contrairement à la pratique retracée jusqu'à présent, selon laquelle les citations occupent la première place, ce document témoigne d'un élan critique plus déductif, qui formule a priori une série d'abstractions propre à chaque ouvrage et qui, par conséquent, maintient entre eux des divisions très strictes. Un autre document pareillement schématique rassemble en sept blocs une collection de termes encore plus abstraits, dont le premier bloc est «L'ennui l'absence de pensée - l'imprécision - l'irrésolution - l'indéfini - le vague ${ }^{20}$ ». Certains mots sont soulignés en rouge, d'autres encerclés en bleu, d'autres encore ajoutés postérieurement en bleu. Encore une fois, l'intention précise de ce document reste difficile à saisir, mais sa simple existence fait ressortir une pensée critique qui s'oriente autour d'un attirail de concepts structurants présélectionnés. L'établissement d'un réseau d'idées jugées pertinentes pour Flaubert mène à la composition d'une armature conceptuelle qui trie les citations amassées. Ces deux feuillets nuancent la compréhension de la critique d'identification, exigeant d'être prise en compte au carrefour de deux volontés, ou plutôt de deux gestes critiques : le premier, inductif, qui amasse des citations et

14. Georges Poulet, Études sur le temps humain, [1949], Edinburgh, Edinburgh University Press, Paris, Librairie Plon, 1950, p. 308.

15. Ibid.

16. Ibid., p. 309.

17. Dans sa préface aux Métamorphoses du cercle, Jean Starobinski affirme l'intégralité de cette prédilection: «Chez Poulet, le choix ne consiste pas à opérer une discrimination entre les œuvres » (p. 14).

18. Comme Pierre-Marc de Biasi l'a montré dans «Qu'est-ce qu'un brouillon? Le cas Flaubert : essai de typologie fonctionnelle des documents de genèse », dans Pourquoi la critique génétique? : méthodes, théories, dirigé par Michel Contat et Daniel Ferrer, Paris, CNRS, 1998, p. 35, l'établissement d'une définition de l'avant-texte n'est pas du tout une certitude. La difficulté concerne, en particulier, l'étroitesse relative avec laquelle on utilise le terme, car «elle peut aussi bien désigner au sens strict les documents exclusivement relatifs à la fonction de textualisation, ou, en un sens un peu plus large, un ensemble comprenant à la fois ces documents et les manuscrits de travail relatifs à d'autres fonctions (structuration, documentation) mais qui ont été utilisés pour cette textualisation (plans, scénarios, notes documentaires, etc.)».

19. Fonds Georges Poulet, Boîte 4, A-1-40.

20. Ibid. 
déconstruit les frontières entre les ouvrages visés, le second, déductif, qui façonne une collection de concepts fondamentaux et charpente les divisions entre les mêmes ouvrages.

Une révision tout aussi significative se manifeste si l'on élargit encore davantage la définition de l'avant-texte, en admettant comme donnée signifiante la simple existence d'un document ou dossier dans une archive. Pour nos fins, ce procédé permet de complexifier la représentation commune de Poulet comme critique forcément franco-centré, qui exclut de son corpus d'autres traditions littéraires ${ }^{21}$. Prendre en considération uniquement les avant-textes principaux de ses ouvrages publiés corrobore naturellement cette image, mais si l'on jette un coup d'œil sur ses autres manuscrits, elle se trouve déstabilisée. Certains dossiers concernant Virginia Woolf et Herman Melville se mélangent, en effet, avec ceux concernant Flaubert et Proust, la séparation des dossiers «Cercles» en «Allemagne», «pensée anglaise» et «pensée française » témoignant d'une vision véritablement globale, ou au moins occidentale. Le fait que l'intérêt de Poulet a dépassé les frontières nationales et linguistiques est confirmé par Stéphanie Cudré-Mauroux quand elle fait l'éloge de ses lectures «encyclopédiques » et transnationales ${ }^{22}$. En même temps, le fait que la plupart des fruits de ces lectures sont restés à l'état d'avant-texte signale une intention curieuse de diminuer leur présence lors de chaque rédaction, ce qui aboutit au francocentrisme de l'œuvre publiée.

Ayant schématisé la procédure par laquelle Poulet compose ses ouvrages et ayant profité de ce modèle préliminaire pour clarifier et nuancer ses principes critiques, retournons à la question méthodologique de la pertinence et de la permissivité d'une génétique de la critique. En principe, la génétique porte un regard également minutieux sur les avant-textes de Poulet et de Mallarmé, notamment sur la distinction entre une écriture à la main et celle avec une machine, sur le type d'ajouts et biffures employés, sur la présence relative des notes de régie. Concernant une telle analyse, le jeu en vaut la chandelle indépendamment du genre de l'ouvrage en question. Pourtant, comme l'attestent les feuillets et les dossiers de Poulet, certaines spécificités restent propres à l'analyse génétique de la critique. Elle bénéficie, par exemple, de l'acte d'opérer à une échelle plus vaste, de considérer comme une évidence la simple existence de certains documents ou dossiers, ainsi que leur inclusion ou suppression éventuelle dans l'œuvre publiée. Une telle approche pourrait être effectuée avec Mallarmé, néanmoins elle est plus raisonnable et féconde quand les interprétations prévues concernent une pratique ou un propos général, plutôt que les nuances d'un seul vers.

Cette divergence subtile se manifeste dans une autre spécificité, qui justifie et particularise à la fois la génétique de Poulet : l'envergure de l'aspect visuel de ses avant-textes. Tout aussi charpentés que bariolés, ils accrochent l'œil et exigent d'effectuer un examen presque formaliste pour démêler le sens de leurs ajouts respectifs. Cette caractéristique rappelle et même radicalise la distinction que Louis Hay a faite entre le livre et le manuscrit, quant à la composition hétérogène «de signes graphiques, lettres et mots mais aussi ratures, marques de position (renvois, insertions, déplacements), symboles, sigles, dessins », ce qui en fait un objet visuel qui est perçu au lieu d'être simplement $1 u^{23}$. On pourrait dire qu'une génétique de Poulet s'autorise d'elle-même, car dans les phases prérédactionnelle et rédactionnelle, tout avant-texte n'a pas encore acquis sa distinction formelle. Concernant le genre, le brouillon d'un poème de Mallarmé et celui d'une monographie de Poulet resteront englobés dans le statut d'objet visuel, jusqu'à ce qu'ils basculent dans la phase éditoriale et adoptent chacun leur forme définitive. Néanmoins, il serait peu judicieux d'assimiler entièrement le caractère visuel des deux types d'avant-textes. Le codage et le chiffrage de Poulet attestent, après tout, d'un procédé très systématisé qui pourrait être indubitablement employé par n'importe quel écrivain, mais qui reste plus propre à l'esprit et la pratique critique. Pour prolonger la métaphore de Hay, si les brouillons se présentaient comme des tableaux, la peinture de Poulet serait guidée par des principes doctrinaires, par un système codifié. Le caractère visuel de ses brouillons, par conséquent, corrobore la légitimité de la génétique d'un ouvrage

21. Voir «Intervention lors d'un colloque consacré aux langages des études humanistes » par Éric Weil pour un reproche de ce type concernant le corpus de textes jugés canoniques et que Poulet a choisis. Essais sur la philosophie, la démocratie et l'éducation, Cahiers Éric Weil, IV, Lille, Presses Universitaires de Lille, 1993, p. 175-181.

22. Stéphanie Cudré-Mauroux, «Critiquer, c'est se souvenir», Euvres et critiques, vol. XVIII, n 2, 2002, p. 109.

23. Louis Hay, «Qu'est-ce que la critique génétique?», ITEM, mis en ligne le 9 décembre 2008, <www.item.ens.fr/index.php?id=384032> (consulté en février 2018).. 
critique, tout en soulignant une différence subtile quant aux spécificités de cette visualité.

Outre la proximité matérielle entre ses avant-textes et les supports jugés appropriés pour une analyse génétique, la façon dont Poulet examine lui-même les brouillons de Flaubert révèle une justification encore plus rigoureuse et normative. Dans le chapitre que Poulet a écrit sur l'écrivain normand dans Les Métamorphoses du cercle, son penchant émerge derechef pour mettre en équivalence les citations et les données arrachées aux divers textes. Cependant, cette fois-ci Poulet inclut dans l'amas de supports les brouillons de Madame Bovary, leur octroyant, en effet, la même autorité comme preuve qu'il accorde à l'ouvrage définitif. Il récrimine, par exemple, contre certains choix éditoriaux faits par Flaubert, faisant référence à un passage que «Flaubert n'a malheureusement pas gardé dans la version définitive» et constatant que l'image de «la goutte de vin rouge que l'on laisse tomber dans un verre d'eau », ne devrait pas être remplacée dans la version finale par «une auréole 24 ». L'absence radicale de frontière perçue entre l'avant-texte et le texte mène à un commentaire qui frôle la réécriture, qui recompose l'ouvrage définitif en l'entrelaçant avec ses brouillons. Poulet justifie et même théorise cet acte plus tard dans sa carrière par le rejet d'une deuxième frontière : celle qui existe entre le texte et le critique, entre l'objet visé et l'outil analytique qui le vise ${ }^{25}$. En définissant la critique d'identification comme l'assimilation du Lebenswelt de l'écrivain en question à celui du critique, Poulet enlève la distinction générique entre les produits littéraires réalisés par ces deux figures. Au sein de sa pensée se tapit ainsi la justification inhérente et rigoureuse de la génétique de ses ouvrages. Si l'on intègre l'équivalence que Poulet constate entre Madame Bovary et Les Métamorphoses du cercle avec son propre usage des brouillons de Madame Bovary, on achève une autorisation vraiment rigoureuse de l'usage des brouillons des Métamorphoses du cercle, une solution radicale apportée au scrupule méthodologique qui a lancé et constitué l'objectif de cette étude.

En prenant comme point de départ ce scrupule, on a abordé l'étude de cas de Georges Poulet en se focalisant sur un dossier d'avant-textes qu'il a rédigé à propos de Gustave Flaubert. Travaillant de façon inductive avec les subtilités d'un échantillon de documents, on a cherché à codifier les trois étapes principales qui constituent sa procédure critique. Ensuite, on a relié ce schéma à certains penchants propres à une critique d'identification telle que Poulet l'a théorisée. Finalement, on a abouti à quelques réflexions plus théoriques à propos de la génétique de la critique, cherchant sa justification et sa spécification dans la pensée de Poulet lui-même. Ayant visé les germes d'une autorisation, posons-nous la question suivante : pourrait-elle fournir un socle théorique pour une génétique de la critique plus générale et codifiée ? Certes, une affinité inhérente existe entre la critique d'identification et la critique génétique, car les deux, situées toujours en opposition au structuralisme, ne croient pas que le texte se réalise dans le temps zéro de sa parution, mais plutôt tout au long de la durée de sa conception 26 . Pourtant, le fait d'accepter, dans un sens plus vaste, tous les propos méthodologiques de Poulet, exigerait une révision assez radicale de l'aboutissement recherché dans une critique génétique. Dans ce cas, il ne s'agirait ni de l'achèvement d'un texte englobant toutes les étapes de rédaction, typique d'une édition génétique, ni de l'usage de cette édition pour des démarches herméneutiques, mais de l'accès et de l'approfondissement du Lebenswelt composé par les germes, l'évolution, et la parution de l'ouvrage.

Avançons ainsi vers une possibilité à la fois plus raisonnable et plus radicale : ne pas postuler une seule génétique de la critique, mais penser plutôt une multiplicité, chaque enquête portant une cohérence avec l'esprit du critique

24. Georges Poulet, Les Métamorphoses du cercle, Paris, Librairie Plon, 1961, p. 398.

25. Plusieurs formulations de cette idée se trouvent dans La Conscience critique, un ouvrage dans lequel Poulet retrace l'évolution de diverses pratiques critiques tout en codifiant sa propre méthode. Cette méthode est fondée sur la mise en équivalence des tentatives du poète et du critique : «Le seul bon critique est le critique-poète, celui qui pour remplir ses fonctions fait appel en lui-même à des ressources proprement poétiques. Son devoir est de trouver au poème un équivalent qui rivalise de poésie avec celui-ci» (p. 46). Plus tard dans le texte, il extrapole cette similitude à toutes les formes littéraires : «Tout texte littéraire, essai, roman ou poème, avait aussi son point de départ [...] toute littérature était philosophie, toute philosophie était littérature» (p. 306).

26. Voir Éléments de critique génétique, où Almuth Grésillon retrace la naissance et le développement de la discipline, tout en alléguant qu'elle est apparue dans les années soixante-dix explicitement contre le structuralisme qui prend l'ouvrage final comme une singularité synchronique dont le sens s'exprime dans les relations internes du langage. Éléments de critique génétique. Lire les manuscrits modernes, 1994, rééd. Paris, CNRS Éditions, 2016. 
en question. Différencier les études génétiques de cette façon ajouterait un mandat de mimétisme, une exigence d'imitation par rapport au cadre théorique de l'œuvre sondée. Altération notable, elle semble entièrement juste par rapport au projet critique, un projet où chacun façonne son système conceptuel et postule, intrinsèquement, que ce système est capable d'englober d'autres démarches. Au moment où l'objet et le dispositif d'étude se mélangent, au moment où la génétique devient une méta-pratique, il convient de prendre en compte ces volontés et de chercher à adopter une perspective organique par rapport à elles. Dans cette multiplication, dans cette spécification, avec un peu de chance, peut-être trouverons-nous une définition flexible, la base profondément théorique de la critique génétique de la critique 27 .

27. Je remercie chaleureusement Claudia Fontu pour son aide et sa relecture attentive.

COLTON VALENTine est un étudiant en Master 2 du programme Théorie de la Littérature, cohabilité par l'École normale supérieure, l'université Sorbonne Paris IV, et l'École des hautes études en sciences sociales. Originaire de San Francisco, il a obtenu une licence en littérature comparée à Harvard College en 2016, après quoi il a commencé ses études en France grâce à la bourse William-Lodge. Ses travaux se focalisent sur les littératures française, anglaise et allemande des XIXe et XXe siècles, ainsi que sur la critique littéraire du XXe siècle; ses intérêts de recherche portent sur le champ littéraire transnational, l'intertextualité, la temporalité et les enjeux fluctuants de la mimesis. 


\section{Résumés \\ Vers une génétique de la critique : le cas de Georges Poulet}

Traditionnellement, la critique génétique retrace les métamorphoses d'un ouvrage littéraire, voire poétique ou fictif, à travers ses brouillons et ses variantes publiées. Dans «Vers une génétique de la critique : le cas de Georges Poulet», on s'interroge sur les conséquences d'une adaptation de cette méthode aux ouvrages de la critique littéraire. À cette fin, on aborde l'étude du cas de Georges Poulet, l'écrivain belge connu pour ses Études sur le temps humain. En s'appuyant sur la richesse des archives au fonds Georges Poulet à Berne, on cherche, premièrement, à codifier les étapes de la «procédure critique» de Poulet : le recueil de citations, leur catégorisation thématique et leur intégration dans l'écriture du critique. Ensuite, on constate que cette procédure soutient et explique certains principes idiosyncratiques de sa «critique d'identification», comme, par exemple, le retrait du contexte de la citation et des distinctions génériques, ainsi que la supposition que chaque œuvre est unifiée de façon organique. Finalement, on explore la façon dont cette praxis elle-même autorise une telle approche génétique de l'œuvre de Poulet. En conclusion, on réfléchit à la mesure dans laquelle ce cas pourrait fournir un socle théorique pour d'autres études de ce genre - et on se demande si une «génétique de la critique» ajoute par définition un mandat de mimétisme : une exigence d'imitation par rapport au cadre théorique de l'œuvre sondée.

In this article I explore the implications of applying textual genetics to works of literary criticism. To do so, I take up the case study of Georges Poulet, the Belgian writer best known for his Études sur le temps humain (Studies in Human Time). Drawing on the rich archives at the Fonds Georges Poulet in Bern, I first seek to codify the stages of Poulet's critical process: the assemblage of quotations, their thematic categorization, and their integration in the critic's writing. Next, I claim that this systemic procedure undergirds certain idiosyncratic principles of Poulet's "criticism of identification" such as the elision of quotation context and of generic distinctions, as well as the presumption that every author's œuvre is organically unified. I then explore the way this unusual praxis itself authorizes a genetic critical approach to Poulet's work. In closing, I reflect on the extent to which Poulet's case might offer a theoretical basis for other similar studies - and whether a "génétique de la critique" (a "genetics of criticism") must, by definition, operate mimetically: by imitating the theoretical framework of the critical work in question.

In „Vers une génétique de la critique: le cas de Georges Poulet“ fragen wir uns, welche Konsequenzen die Anpassung der genetischen Methode an die Werke der Literaturkritik hat. Zu diesem Zweck diskutieren wir den Fall von Georges Poulet, einem belgischen Literaturkritiker, der für seine Études sur le temps humain bekannt ist. Auf der Basis der Materialfülle des Archivs im Fonds Georges Poulet in Bern versuchen wir zunächst, die Schritte der ,procédure critique“ von Poulet systematisch zu erfassen: durch das Sammeln von Zitaten, deren thematische Kategorisierung und die Integration in das Schreiben der Literaturkritik. Dann stellen wir fest, dass dieses Verfahren einige eigenwillige Prinzipien seiner ,,critique d'identification“ bestärkt und erklärt, wie zum Beispiel den Wegfall des Kontexts des Zitats und seiner Gattungsunterschiede sowie die Annahme, dass jedes Werk auf organische Weise zu seiner Einheit findet. Schließlich untersuchen wir, inwiefern diese Praxis zu einer genetischen Annäherung an die Arbeit von Poulet Anlass gibt. In fine fragen wir uns, ob dieser Fall eine theoretische Grundlage für weitere Studien dieser Art bieten könnte - und ob eine ,génétique de la critique“ per definitionem die Notwendigkeit einer Mimesis mit sich bringt: indem sie nämlich den theoretischen Rahmen des untersuchten Werks nachahmt.

Este artículo abre un interrogante acerca de las consecuencias de una adaptación del método genético a las obras de crítica literaria. Con este fin, se aborda el estudio del caso de Georges Poulet, escritor belga conocido por sus Études sur le temps humain. Aprovechando la riqueza de los archivos en el Fondo Georges Poulet de Berna, se intenta, primero, codificar las etapas del "procedimiento crítico" de Poulet: la recopilación de citas, su categorización temática y su integración en la escritura del crítico. Luego, se constata que este procedimiento sostiene y explica ciertos principios idiosincrásicos de su "crítica de identificación", como, por ejemplo, la omisión del contexto de la cita o de las distinciones genéricas, así como la suposición de que cada obra está unificada de manera orgánica. Finalmente, se explora la manera en que esta praxis permite un enfoque genético de la obra de Poulet, para preguntarse, a modo de conclusión, si este caso puede brindar una base teórica para otros estudios de este tipo, y si una "genética de la crítica" comporta un mandato de mimetismo: una exigencia de imitación con respecto al marco teórico de la obra analizada.

Este artigo interroga-se sobre as consequências de aplicar o método genético a obras de crítica literária, estudando para isso o caso de Georges Poulet, escritor belga, conhecido pelos Études sur le temps humain. Recorrendo ao seu rico espólio, depositado em Berna, é possível reconstituir as etapas do seu « processo crítico »: a recolha de citações, a sua categorização temática e a sua integração na redacção da crítica. Este processo ajuda a compreender alguns princípios idiossincráticos da sua « crítica de identificação », tais como, por exemplo, a descontextualização da citação e das distinções genéricas, ou como a convicção de que cada obrao é unificada de forma orgânica. Finalmente, explora-se como esta práxis autoriza que a obra de Poulet receba uma abordagem genética. Em conclusão, pergunta-se se este caso poderia fornecer uma base teórica para mais estudos do mesmo tipo - e se uma « genética da crítica » comporta um mandato intrínseco de mimetismo: uma exigência de imitação face ao quadro teórico da obra pesquisada.

L'articolo s'interroga sulle conseguenze di un adattamento del metodo genetico ai testi di critica letteraria, prendendo in esame il caso di Georges Poulet, critico belga noto per i suoi Études sur le temps humain. Basandosi sui ricchi archivi del fondo Georges Poulet di Berna, si cerca, innanzitutto, di codificare le fasi della "procedura critica" di Poulet: la raccolta di citazioni, la loro categorizzazione tematica e la loro integrazione nella scrittura del critico. In seguito, si constata come questa procedura sostenga e spieghi alcuni principi idiosincratici della sua "critica d'identificazione", quali il ritiro dal contesto della citazione e delle distinzioni generiche, o l'ipotesi che ogni opera sia organicamente "unificata". Infine, si esplora il modo in cui questa praxis autorizza un approccio genetico dell'opera di Poulet. In conclusione, ci si chiede se questo caso possa fornire una base teorica per altri studi di questo tipo - e se una "genetica della critica" possa funzionare, per definizione, in maniera mimetica: imitando il quadro teorico del testo critico in questione. 